\title{
GPIIb/IIIa Receptor Antagonism Using Small Molecules Provides no Additive Long-Term Protection after Percutaneous Coronary Intervention as Compared to Clopidogrel Plus Aspirin
}

\author{
Michele Schiariti ${ }^{1,2}$, Angela Saladini ${ }^{1}$, Francesco Papalia ${ }^{2}$, Placido Grillo ${ }^{1}$, Cristina Nesta ${ }^{1}$, \\ Domenico Cuturello ${ }^{2}$, Bindo Missiroli ${ }^{1}$ and Paolo Emilio Puddu ${ }^{2, *}$

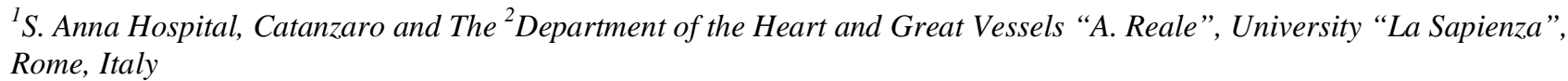

Abstract: Background: There is some controversy as to whether tirofiban or eptifibatide, two small anti-aggregating drugs (AAD), may reduce the incidence of composite ischemic events within one year in patients undergoing percutaneous coronary intervention (PCI) in the real clinical world.

Methods: We compared consecutive patients on oral double AAD (with clopidogrel and aspirin) who underwent PCI $(n=207)$ and patients who were on single AAD and received a second AAD, just prior to PCI, and either high-dose tirofiban or double-bolus eptifibatide (double AAD plus small molecules group, $n=666$ ). The primary end point (incidence of composite ischemic events within one year) included death, acute myocardial infarction, unstable angina, stent thrombosis or repeat PCI or coronary bypass surgery (related to the target vessel PCI failure) and was modelled by Cox's regression.

Results: There were 89 composite ischemic events: 24 (11.6\%) in double AAD alone and 65 (9.8\%) in double AAD plus small molecules groups (log-rank test: $\mathrm{p}=0.36$ ). Incidences by type of ischemic events were similar between the 2 groups. Based on 21 potential covariates fitted simultaneously, adjusted hazard ratios (HR and 95\% confidence intervals) showed that age (HR 1.03, 1.01-1.06, p=0.01), diabetes (HR 1.68, 1.01-2.79, $\mathrm{p}=0.05)$ and intra aortic balloon pump (HR 5.12, 2.36-11.10, $\mathrm{p}=0.0001)$ were significant risk factors whereas thrombolysis by tenecteplase $(\mathrm{HR} 0.35,0.13-0.98, \mathrm{p}=0.05)$ and having had hypertension or anti-hypertensive treatment (HR 0.58, 0.36-0.93, $\mathrm{p}=0.03$ ) were significant protectors for events. Whether small molecules were present provided a non significant additional benefit as compared to double AAD alone (HR 0.83, 0.51-1.36, p=0.46). Pre-PCI CK-MB were not useful to predict events (HR 1.01, 0.99-1.01, p=0.17).

Conclusions: In clinical world patients undergoing PCI (rescue plus primary <13\%) while on double AAD, based on clopidogrel plus aspirin, small molecules (tirofiban or eptifibatide) provided no additive long-term protection against the occurrence of composite ischemic events whereas thrombolysis by tenecteplase did.

Keywords: GP IIb/IIIa; tirofiban high-dose; eptifibatide double-bolus; clopidogrel; aspirin; tenecteplase; Thrombolysis; double anti-aggregation; ischemic events; PCI.

\section{INTRODUCTION}

Although glycoprotein (GP) IIb/IIIa antagonists are effective anti aggregating drugs (AAD) in acute coronary syndromes (ACS) after percutaneous coronary intervention (PCI) [1], the optimal timing to start these agents in unstable angina or non-ST elevation myocardial infarction (NSTEMI) and the optimal doses and type of agent have not been defined [2, 3]. In ST elevation myocardial infarction (STEMI) patients, much of the evidence favouring the use of GP IIb/IIIa antagonists was obtained in the era before dual oral antiplatelet therapy and largely by placebo-controlled comparisons [3]. For those patients with STEMI who present

\footnotetext{
*Address correspondence to this author at the Dipartimento del Cuore e Grossi Vasi "Attilio Reale", UOC Biotecnologie Applicate alle Malattie Cardiovascolari, Università degli Studi di Roma "La Sapienza", Viale del Policlinico, 155, Rome 00161, Italy; Tel: +39.06.4455291;

Fax: +39.06.4441600; E-mail: paoloemilio.puddu@uniroma1.it
}

to hospitals that do not perform PCI, fibrinolysis is still achieved, which was associated with significantly fewer 30day ischemic complications if PCI was performed within the successive 6 hours [4].

In ACS patients treated by PCI, there are several studies assessing the efficacy of eptifibatide versus placebo or abciximab [5-7] and of abciximab versus high-dose tirofiban [8-10]. In STEMI or diabetic patients, meta-analyses were performed $[11,12]$ as the idea that a class effect might be present has emerged with accompanying pharmacoeconomic consequences [13-15]. In a single-centre study a long-term survival advantage with tirofiban, followed by eptifibatide and then abciximab was observed [7]. However, among STEMI patients, a substantial similarity between tirofiban and abciximab was seen in terms of in-hospital safety and 30-day efficacy [10]. On the other hand, the incidence of composite ischemic events have been rarely investigated $[5,7,10]$ and none considered standard therapy 
with double AAD based on clopidogrel and aspirin versus small molecules as a group.

The Sant'ANna TIrofiban Safety Study (SANTISS: www.clinicaltrials.gov Identifier: NCT00566891) compared the combination of bleeding and access site complications of consecutive, unselected patients undergoing PCI under double AAD regimen, in absence or presence of intravenous high-dose tirofiban given just prior to PCI (down-stream). Between 2004 and 2008, 2205 patients undergoing PCI were included in the SANTISS registry [16]. Between 2005 and early 2007 a block randomised comparison of high-dose tirofiban versus double-bolus eptifibatide [17] was performed among patients undergoing PCI while on double AAD: the incidence of ischemic events within one year was the primary end-point, which is reported here. Thus patients who underwent PCI on double AAD with clopidogrel and aspirin during the randomised phase of SANTISS are compared to patients treated with the additive use of GPIIb/IIIa antagonistic small molecules tirofiban or eptifibatide, considered as a group. Pre-PCI levels of creatinekinase myocardial band (CK-MB) were measured since: a) small molecules may affect them, b) this may impact on long-term ischemic event incidence [18], and c) there is a close relation between peri-procedural myocardial infarct and one year mortality [19].

\section{MATERIALS AND METHODS}

All patients at Sant'Anna Hospital, Catanzaro, who underwent elective, primary or rescue PCI were eligible to be enrolled in SANTISS [16]. Patients referred from another hospital with ongoing tirofiban or eptifibatide treatment were excluded from this study. A block randomisation scheme was selected to fit the ongoing progress of SANTISS and the power calculated based on the following: a) a 3 to $6 \%$ absolute one year survival difference between treatments during a 3 -year accrual time; b) a 3 to 4 ratio between tirofiban- and eptifibatide-enrolled patients; c) a maximum of $2 \%$ patients lost to follow-up; and d) a log-rank test power analysis with alpha equal to $5 \%$ and power greater than $80 \%$. Accordingly, 675 consecutive patients were block-randomized: there were 75 blocks of 4 and 75 blocks of 5 patients, each having one patient randomized to eptifibatide and the remaining to tirofiban.

Patients who were on oral single antiplatelet regimen (ASA or clopidogrel) received a down-stream open-label GPIIb/IIIa small molecule (tirofiban or eptifibatide) and a second oral antiplatelet agent was added immediately before PCI. When the first AAD was clopidogrel, the second agent was ASA (at the loading dose of 160-325 mg orally and then 80-125 mg orally indefinitely). If the first antiplatelet agent was ASA, we gave clopidogrel as the second agent (at the loading dose of $300 \mathrm{mg}$ orally and then $75 \mathrm{mg} / \mathrm{d}$ for at least 3 months). Patients who already were on oral double AAD regimen (ASA at $80-125 \mathrm{mg} / \mathrm{d}$ plus clopidogrel $75 \mathrm{mg} / \mathrm{d}$ ) were considered as a separate group. Patients who were on oral ASA plus ticlopidine or who had ticlopidine as the first AAD were excluded. A few patients in whom a second oral antiplatelet drug could not be used were also excluded [16].

GPIIb/IIIa small molecules were given as follows: a) high-dose tirofiban [16]: $25 \mu \mathrm{g} / \mathrm{Kg}$ bolus followed by 0.15 $\mu \mathrm{g} / \mathrm{Kg} / \mathrm{min}$ 18-hour infusion; b) double-bolus eptifibatide
[17]: $180 \mu \mathrm{g} / \mathrm{Kg}$ bolus, followed by $2 \mu \mathrm{g} / \mathrm{Kg} / \mathrm{min} 18$-hour infusion, and $10 \mathrm{~min}$ after a second $180 \mu \mathrm{g} / \mathrm{Kg}$ bolus.

For the purpose of the present analysis the time frame of data collection was from PCI to one year after. The primary end-point was the combination of ischemic events: cardiac death, acute myocardial infarction, unstable angina, acute stent thrombosis or the need for repeat PCI or coronary bypass surgery (related to the target vessel PCI failure), all associated with a date but with identical rank order. When more than 1 event occurred only the first one was considered. An external reviewer (PEP), unaware of treatment assignment, coded all events. Subgroups of patients referred to perform a rescue-PCI within 6 hours after thrombolysis were included.

All patients underwent pre- and post-procedural standard electrocardiograms (ECG). CK-MB was routinely measured at admission in hospital as well as 6 and 12 hours after the procedure and before discharge. Q-wave myocardial infarction was diagnosed when new $\mathrm{Q}$ waves appeared at least in two leads with an increase in CK-MB at least twice upper the normal limit (core laboratory upper limit: $15 \mathrm{U} / \mathrm{l}$ ) was observed. Non-Q-wave myocardial infarction was diagnosed when an increase in CK-MB at least threefold upper the normal limit was observed.

Interventions were performed by 6- or 7-F guiding catheter using the femoral approach. Operators were free to choose the more adequate catheter curves, guidewires and stents whose type (drug-eluting versus bare-metal), length, diameter, and number were coded but are not considered for the purpose of the present analysis. A bolus of heparin (70 $\mathrm{UI} / \mathrm{kg}$ ) was given before starting the procedure in order to maintain an activated clotting time (ACT) of $250 \mathrm{~s}$; further doses of heparin were given to adjust ACT when necessary.

Data obtained from a dedicated data form for SANTISS by means of a purposely compiled (by DC) software facilitated the automatic transfer from records on paper to an Excel file which was analysed by BMDP statistical package after transformation into a machine readable file. Summary statistics of continuous variables were expressed as mean value \pm SD whereas discrete variables were given only as proportions. T-test was used to assess inter-group differences. Log-rank test was used to test the univariate intergroup significance in the incidence (Kaplan-Meier curves) of composite events during 365 days of follow-up. Moreover, the presence (coded 1) or absence (coded 0) of composite events was modelled by using Cox's proportional hazards model, an exponential model of survival (Y) prediction whereby it is easy to obtain the probability of an event function (1-Y) given by: $1-\mathrm{Y}=\mathrm{S}(0)$ exponent of coefficient A1 $(\mathrm{x} 1-\mathrm{X} 1) *$ exponent of coefficient An (xn-Xn), which is calculated based on the cumulative proportional hazard $(\mathrm{S}(0))$ and on the coefficients of covariates (A1 to An), which stay in the model solution. All covariates were in at step zero (forced method) in order to compute their individual relative contribution. Further details are given elsewhere [20]. NCSS software version 2007 (released August 14, 2007 by J Hintze, Kaysville, Utah; see www.ncss.com) was used and Wald's t values and probability level, along with hazard ratios (HR) and $\pm 95 \%$ confidence intervals (CI) were com- 
puted [20-22]. A value of $p<0.05$ was considered statistically significant.

\section{RESULTS}

Of the 885 consecutive patients who underwent elective PCI at Sant'Anna hospital or were sent for primary or rescue PCI between February 2005 and March 2007, while SANTISS was ongoing [16], 525 were block-randomized (3.5:1) to tirofiban and 150 to eptifibatide. There were $12(1.4 \%)$ patients who were lost to follow-up: $6(1.1 \%)$ in the tirofiban and $3(2 \%)$ in the eptifibatide group and $3(1.4 \%)$ in the double AAD alone group. There were therefore 666 patients in the double AAD plus GPIIa/IIIb small molecules group and 207 patients in the double AAD alone group.

There were 89 composite ischemic events within one year, including death, acute myocardial infarction, unstable angina, stent thrombosis or repeat PCI or coronary bypass surgery (primary end-point on the present study): 24 (11.6\%) in double AAD alone and $65(9.8 \%)$ in double AAD plus small molecules groups (log-rank test: $p=0.36$, Fig. 1). There was no significant difference in the proportions of specific ischemic events within one year between double AAD patients without or with small molecules: major events (death or acute myocardial infarction) were seen respectively in 10 $(4.8 \%)$ and $17(2.6 \%)$ patients, minor events were respectively $8(3.9 \%)$ and $20(3.0 \%)$ for unstable angina, $2(1.0 \%)$ and $7(0.6 \%)$ for stent thrombosis and $4(1.9 \%)$ and 21 (3.1\%) for repeat PCI or coronary bypass surgery. The hazard rate presentation was superposable between the groups (Fig. 2). There were only 9 of $873(1.0 \%)$ patients who presented with multiple events and 8 were seen in the tirofiban group.

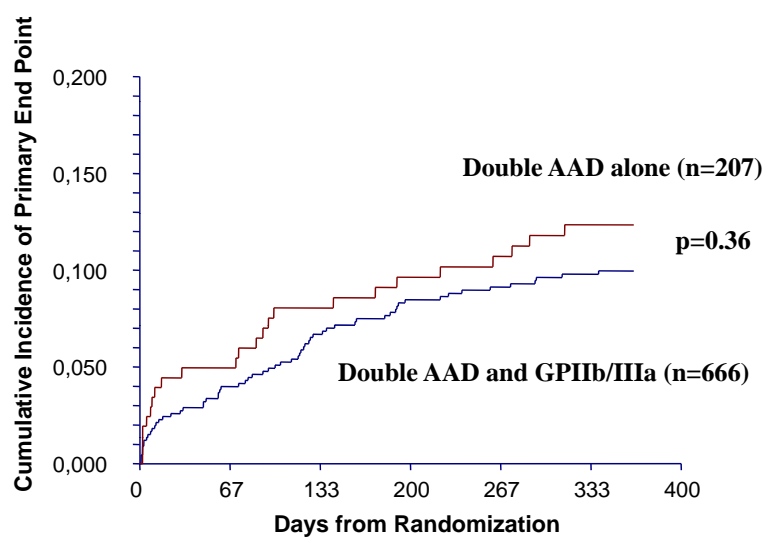

Fig. (1). Kaplan-Meier curves show non significant (by log-rank test) difference between the cumulative incidences of composite ischemic events in double anti-aggregating drug (AAD) alone group versus double AAD plus glycoprotein (GP) IIb/IIIa (eptifibatide or tirofiban) group. The primary end point was the composite of cardiac death, acute myocardial infarction, angina, acute stent thrombosis or the need for repeat percutaneous coronary intervention or coronary bypass surgery, all with identical rank order. When more than one event occurred only the first one was considered.

Table 1 shows the multivariate prediction of composite ischemic events within one year based on 21 potential covariates fitted simultaneously by Cox's model. Adjusted hazard ratios showed that age (HR 1.03, 95\%CI 1.01-1.06, $\mathrm{p}=0.01)$, diabetes $(\mathrm{HR} 1.68,95 \% \mathrm{CI} 1.01-2.79, \mathrm{p}=0.05)$ and intra aortic balloon pump (HR 5.12, 95\% CI 2.36-11.10, $\mathrm{p}=0.0001$ ) were significant risk factors whereas thrombolysis by tenecteplase (HR $0.35,95 \%$ CI $0.13-0.98, p=0.05$ ) and having had hypertension or anti-hypertensive treatment (HR $0.58,95 \%$ CI $0.36-0.93, \mathrm{p}=0.03$ ) were significant protectors for events. Whether small molecules were present provided a non significant additional benefit as compared to double AAD alone (HR 0.83, 95\% CI 0.51-1.36, p=0.46). Pre-PCI CK-MB were not useful to predict events (HR 1.01, 95\% CI $0.99-1.01, p=0.17)$. The remaining covariates had a slightly contributor role but did not reach the statistical level of significance. In particular there was no contribution of the variable coding for the number of treated coronary vessels.

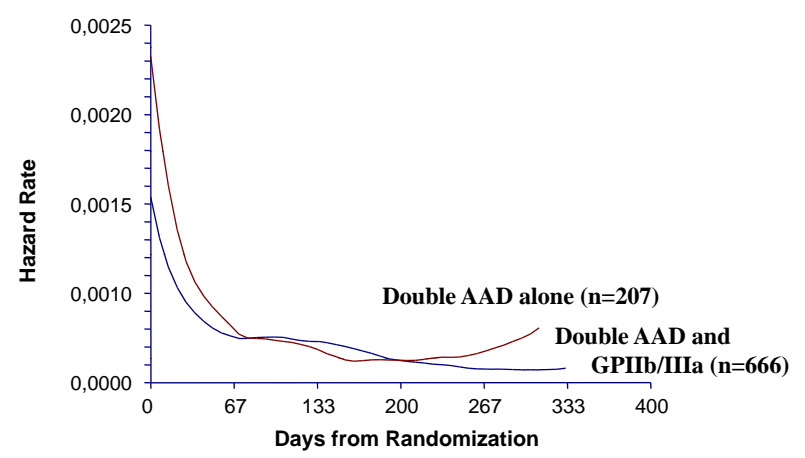

Fig. (2). Comparison between double anti-aggregating drug (AAD) alone group versus double AAD plus glycoprotein (GP) IIb/IIIa (eptifibatide or tirofiban) group show similarly continuous decreasing hazard rate, from immediately after percutaneous coronary intervention to one year afterwards.

\section{DISCUSSION}

We showed that one year composite ischemic events after PCI in real clinical world patients in whom primary or rescue interventions were less than $13 \%$, were not significantly different whether GP IIb/IIIa small molecules were added or not in the context of double AAD with standard $(300 \mathrm{mg})$ clopidogrel and aspirin. Among these patients where pre-PCI CK-MB were not useful to predict events thrombolysis by tenecteplase was a significant protective factor.

As this evidence was obtained with an extended observation period and a robust predictive model was fitted with all potential contributors simultaneously assessed, it is important to see these data in the spotlight of the class-action concepts of small anti-aggregating molecules versus abciximab [7-12, 14, 15], high-dose (600 mg or more) clopidogrel [23] and other drugs such as prasugrel [24, 25] or bivaluridin [26], a series of agents that may actually be considered as optimal treatments in these patients [3]. For all these effectiveness-related comparisons it will be essential to obtain pharmaco-econometric data [13] and/or interaction results with peculiar stenting types [10]. It might be also warranted to consider these results when adopting policy and recommendations existing for patients undergoing PCI [1-3] since recent evidence in patients with STEMI plays against differing too much PCI after thrombolysis [4]. 
Table 1. Multivariable Prediction of Outcome Within One Year Based on 21 Potential Covariates Fitted Simultaneously in 873 PCI Patients

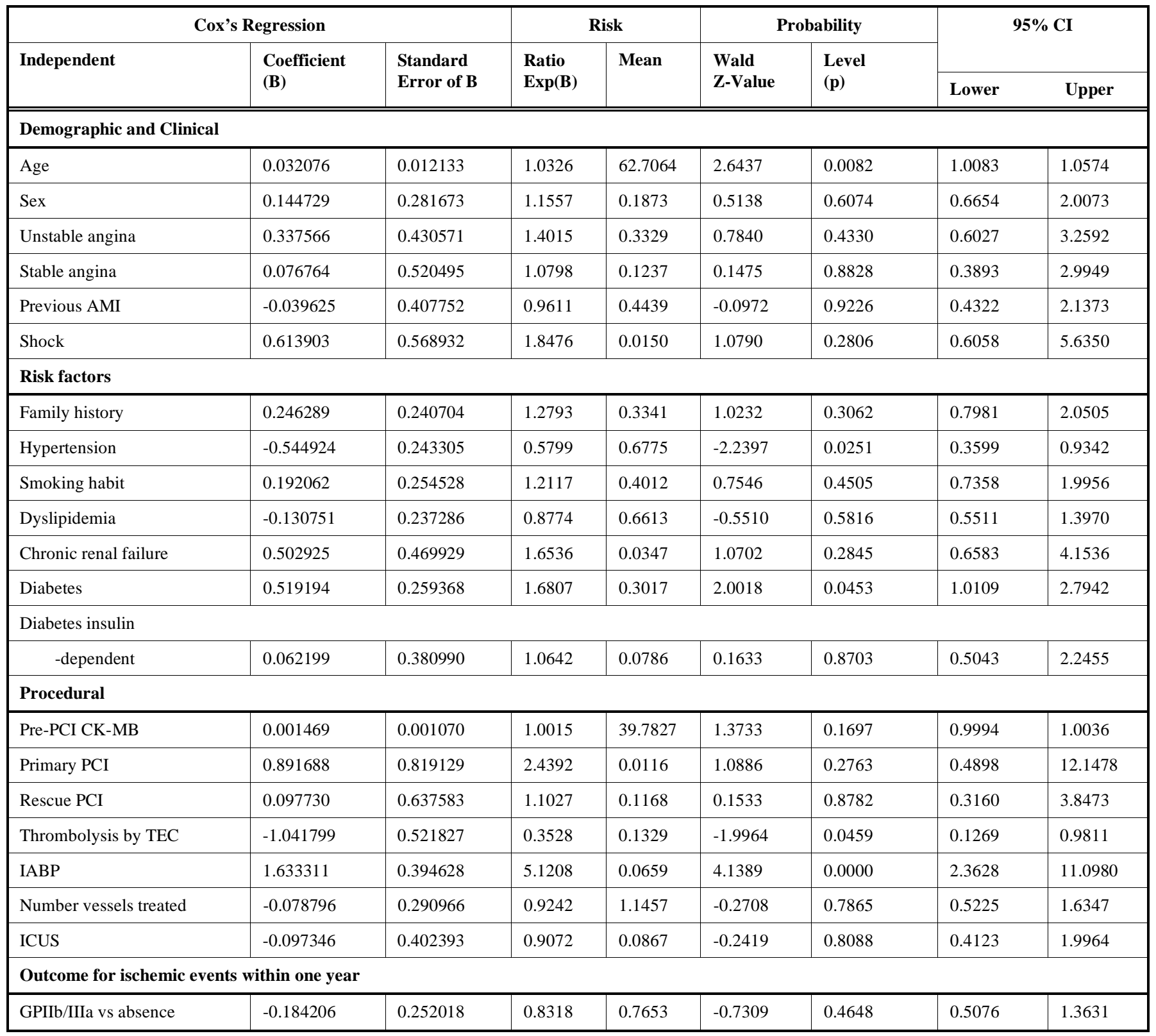

$\mathrm{AMI}=$ acute myocardial infarction; $\mathrm{IABP}=$ intra aortic balloon pump; ICUS = intra coronary ultra sound; PCI= percutaneous coronary intervention; $\mathrm{TEC}=$ tenecteplase; $\mathrm{CI}$ : confidence intervals of relative risk. Note that the model is forced, meaning that all 21 covariates stay in at step zero. Note that the coding of the Grouping variable GPIIb/IIIa vs absence was Double anti-aggregating drug (AAD) treatment only=0; Double AAD and GPIIb/IIIa=1. Thus, in this study where the proportion of Double AAD and GPIIb/IIIa patients was $83 \%$, the multivariate risk of composite ischemic events within one year was not significantly $(\mathrm{p}=0.4648)$ lower (Wald $\mathrm{t}=-0.73$, 95\%CI $0.5076-1.3631$ ) than in those receiving Double AAD only.

One may argue that the no significant modification of long-term outcome seen here was not the consequence of protective failure due to GP IIb/IIIa small molecules but rather to similar efficacy of the two underlying regimens of double AAD, both with clopidogrel and aspirin. GP IIb/IIIa small molecules were given in our study so that their antiaggregating action would be present during the window phase necessary for $300 \mathrm{mg}$ clopidogrel loading dose fully developed its action. On the other hand, those patients who were on clopidogrel $(75 \mathrm{mg} / \mathrm{d})$ were put on double AAD by receiving aspirin loading doses (160-325 mg). When patients presented with double AAD with clopidogrel and aspirin they were not given GPIIb/IIIa small molecules. However, it is important to understand that these latter patients were on clopidogrel $75 \mathrm{mg} / \mathrm{d}$ and aspirin $80-125 \mathrm{mg} / \mathrm{d}$. There are no comparative studies where the long-term efficacy of these pharmacodynamically different double AAD regimens have been assessed and therefore we are unable to dissociate, between our two groups, whether this affected and/or how much our results. Neither could we exclude that higher than $300 \mathrm{mg}$ loading doses of clopidogrel (with or without GP IIb/IIIa small molecules) could be even more effective and a long-term difference would be observed as compared to our double AAD alone patients. Clearly ad hoc studies are needed. 
Lotrionte et al. recently presented a systematic review and meta-analysis of the optimal clopidogrel loading dose in patients scheduled for catheterization and/or PCI in the Literature as of December 2006 [23]. Overall, a high loading dose had an efficacy, also among randomized trials alone, much higher than the standard loading dose: cardiac death or nonfatal myocardial infarction were prevented (odds ratio $0.54,95 \%$ CI $0.32-0.90, \mathrm{p}=0.02$ ), without any statistically significant increase in major or minor bleedings $(\mathrm{p}=0.55$ and $\mathrm{p}=0.98$, respectively). Interestingly, meta-regression disclosed a significant interaction between event rate and the benefits of high loading doses ( $\mathrm{p}=0.005)$, suggesting that the greater the underlying risk, the greater the favourable impact of a high loading dose. Therefore efficacy seemed dosedependent in spite of a dose-independent safety profile. Nevertheless, results over one months of these regimens remain unknown.

There is only one single centre investigation to have evaluated the long-term mortality after administration of abciximab, eptifibatide, and tirofiban during PCI [7]. However, the bolus-only strategy was investigated, based on the idea that it might be similar in efficacy, safer, and more costeffective compared to a bolus plus infusion of GP IIb/IIIa inhibitors. The long-term mortality was not significantly different in the tirofiban group compared to the abciximab group $(\mathrm{p}=0.33)$ or the eptifibatide group $(\mathrm{p}=0.20)$ [7]. We provide support to these data, although it is important to point out that the perfusion strategy we adopted was different as were the definitions of the primary end-point and the observation period.

The relations of elevated CK-MB to clinical outcomes after PCI in patients enrolled in Integrilin to Minimize Platelet Aggregation and Coronary Thrombosis-II (IMPACT-II) trial on eptifibatide was investigated among patients undergoing elective, scheduled PCI for any indication [18]. Parallel analyses investigated CK $(\mathrm{n}=3535)$ and CK-MB $(\mathrm{n}=2341)$ levels after PCI (within 4 to $20 \mathrm{~h})$. Elevated CKMB was associated with an increased risk of death, reinfarction, or emergency revascularization at 30 days, and of death, re-infarction, or surgical revascularization at 6 months. The degree of risk correlated with the rise in CK or CK-MB, even for patients with successful procedures not complicated by abrupt closure [18]. In the present study, prePCI CK-MB was unrelated to outcome.

A very recent meta-analysis [12] of 6 randomized trials on STEMI patients involving 2197 patients showed that abciximab did not reduce 30 -day mortality $(2.2 \%$ vs. $2.0 \%$, p $=0.66)$ or re-infarction $(1.2 \%$ vs. $1.2 \%, \mathrm{p}=0.88)$, nor was there any difference in major bleeding complications $(1.3 \%$ vs. $1.9 \%, p=0.27$ ). Thus, among STEMI patients undergoing primary PCI, similar results between abciximab and small molecules in terms of angiographic, electrocardiographic, and clinical outcome [12] may dictate the selection of the less expensive among these agents [13], especially if a class-effect is present, as quite recently hypothesized $[14,15]$.

More recently, in patients with ACS with scheduled PCI, it was shown that with prasugrel, careful titration might be necessary to obtain a safety profile comparable to an efficacy-equivalent dose of clopidogrel [25]. On the other hand, in STEMI patients undergoing primary PCI with bivalirudin or unfractionated heparin plus a GP IIb/IIIa inhibitor, a $600 \mathrm{mg}$ clopidogrel loading dose was an independent predictor of lower rate of 30-day major adverse cardiac events (HR 0.72, 95\% CI 0.53-0.98), $\mathrm{p}=0.04$ ). Finally, there is evidence that statins exert multiple beneficial effects on patients undergoing PCI [27]. A limitation of our study is that whether statins were given at baseline was not recorded.

In conclusion, patients undergoing PCI (the great majority by elective procedure) while on double antiplatelet therapy (with clopidogrel and aspirin) have an incidence of composite ischemic events within one year which is not significantly different when they also receive GP IIb/IIIa small molecules. It seems that these effects are not related to CK-MB changes. The protective long-term role of thrombolysis was observed as was the higher risk due to intra aortic balloon pump, diabetes and older age. Having had hypertension or anti-hypertensive treatment was a significant protector for events, an observation which fits with epidemiologic data where the paradox of treated hypertension as a protective factor is frequently seen.

\section{REFERENCES}

[1] Topol EJ, Byzova TV, Plow EF: Platelet IIb-IIIa blockers. Lancet 1999; 353: 227-31.

[2] Smith SC Jr, Feldman TE, Hirshfeld JW Jr, et al. American College of Cardiology/American Heart Association Task Force on Practice Guidelines; ACC/AHA/SCAI Writing Committee to Update the 2001 Guidelines for Percutaneous Coronary Intervention: ACC/AHA/SCAI 2005 guideline update for percutaneous coronary intervention: a report of the American College of Cardiology/American Heart association Task Force on Practice Guidelines (ACC/AHA/SCAI writing Committee to update the 2001 Guidelines for percutaneous coronary intervention). J Am Coll Cardiol 2006; 47: e1-e121.

[3] Kushner FG, Hand M, Smith SC Jr, et al.; 2009 Focused Updates: ACC/AHA Guidelines for the Management of Patients With STElevation Myocardial Infarction (Updating the 2004 Guideline and 2007 Focused Update) and ACC/AHA/SCAI Guidelines on Percutaneous Coronary Intervention (Updating the 2005 Guideline and 2007 Focused Update) A Report of the American College of Cardiology Foundation/American Heart Association Task Force on Practice Guidelines. J Am Coll Cardiol 2009; 54: 2205-41.

[4] Cantor WJ, Fitchett D, Borgundvaag B, et al. TRANFER-AMI Trial Investigators: Routine early angioplasty after fibrimolysis for acute myocardial infarction. N Engl J Med 2009; 360: 2705-18.

[5] O'Shea JC, Hafley GE, Greenberg S, et al. ESPRIT Investigators (Enhanced Suppression of the Platelet IIb/IIIa Receptor with Integrilin Therapy trial): Platelet glycoprotein IIb/IIIa integrilin blockade with eptifibatide in coronary stent intervention: the ESPRIT trial. a randomized controlled trial. JAMA 2001; 285: 2468-2473.

[6] Giugliano RP, White JA, Bode Ch, et al.; EARLY ACS Investigators: Early versus delayed, provisional eptifibatide in acute coronary syndromes. N Engl J Med 2009; 360: 2176-90.

[7] Marmur JD, Poludasu S, Lazar J, Cavusoglu E. Long-term mortality after bolus-only administration of abciximab, eptifibatide, or tirofiban during percutaneous coronary intervention. Catheter Cardiovasc Interv 2009; 73: 214-21.

[8] Topol EJ, Moliterno DJ, Herrmann HC, et al.; TARGET Investigators (Do Tirofiban and ReoPro Give Similar Efficacy Trial): Comparison of two glycoproteins IIb/IIIa inhibitors, tirofiban and abciximab, for the prevention of ischemic events with percutaneous coronary revascularization. N Engl J Med 2001; 344: 1888-94.

[9] Bolognese L, Calzini G, Liistro F, et al. Randomized comparison of upstream tirofiban versus downstream high dose tirofiban or abciximab on tissue level perfusion and troponin release in high risk acute coronary syndromes treated with percutaneous coronary interventions: the EVEREST trial. J Am Coll Cardiol 2006; 47: 522-8.

[10] Valgimigli M, Campo G, Percoco G, et al. MULTISTRATEGY Investigators: Comparison of angioplasty with infusion of tirofiban 
or abciximab and with implantation of sirolimus-eluting or uncoated stents for acute myocardial infarction: the MULTISTRATEGY randomized trial. JAMA 2008; 299: 1788-99.

[11] Bhatt DL, Marso SP, Lincoff AM, Wolski KE, Ellis SG, Topol EJ. Abciximab reduces mortality in diabetics following percutaneous coronary intervention. J Am Coll Cardiol 2000; 35; 922-8.

[12] De Luca G, Ucci G, Cassetti E, Marino P. Benefits from small molecule administration as compared with abciximab among patients with ST-segment elevation myocardial infarction treated with primary angioplasty. J Am Coll Cardiol 2009; 53: 1668-73.

[13] Le Pen C, Lilliu H. Choice of GPIIb/IIIa antagonist in percutaneous coronary intervention: how should economic criteria be factored in? Pharm World Sci 2005; 27: 83-91.

[14] Brener SJ. The benefits of platelet glycoprotein IIb/IIIa receptor inhibition during primary percutaneous coronary intervention for ST-segment elevation myocardial infarction: drug-specific or class effect? J Am Coll Cardiol 2009; 53: 1674-6.

[15] Zijlstra F. The benefits and risks of Abciximab, eptifibatide, or tirofiban during PCI: class effects or do they differ? Catheter Cardiovasc Interv 2009; 73: 222-3.

[16] Schiariti M, Saladini A, Missiroli B, et al. Safety of downstream high-dose tirofiban bolus among 1578 patients undergoing percutaneous coronary intervention: the Sant'ANna Tirofiban Safety study. J Cardiovasc Med 2010; 11: 250-9.

[17] Gilchrist IC, O'Shea JC, Kosoglu T, et al. Pharmacodynamics and pharmacokynetics of higher-dose, double bolus eptifibatide in percutaneous coronary intervention. Circulation 2001; 104: 406-11.

[18] Tardiff BE, Califf RM, Tcheng JE, et al. Clinical outcomes after detection of elevated cardiac enzymes in patients undergoing percutaneous intervention. IMPACT-II Investigators. Integrilin (eptifibatide) to Minimize Platelet Aggregation and Coronary Thrombosis-II. J Am Coll Cardiol 1999; 33: 88-96.

[19] Lindsey JB, Marso SP, Pencina M, et al. EVENT Registry Investigators: Prognostic impact of periprocedural bleeding and myocardial infarction after percutaneous coronary intervention in unselected patients: results from the EVENT (Evaluation of Drug-
Eluting Stents and Ischemic Events) registry. J Am Coll Cardiol Intv 2009; 2: 1074-82.

[20] Puddu PE, Brancaccio G, Leacche M, et al. OP-RISK Study Group: Prediction of early and delayed postoperative deaths after coronary artery bypass surgery in Italy. Multivariate prediction based on Cox and logistic models and a chart based on the accelerated failure time model. Ital Heart J 2002; 3: 166-81.

[21] Miller Ch C III, Reardon MJ, Safi HJ: Risk stratification. A practical guide for clinicians. Cambridge: University Press, 2001, pp 1-174.

[22] Menotti A, Puddu PE, Lanti M: Il rischio in Cardiologia: dalla teoria alla pratica. Pavia: Edizioni Internazionali srl, 2004, pp 1189.

[23] Lotrionte M, Biondi-Zoccai GG, Agostoni P, et al. Meta-analysis appraising high clopidogrel loading in patients undergoing percutaneous coronary intervention. Am J Cardiol 2007; 100: 1199-206.

[24] Wiviott SD, Braunwald E, McCabe CH, et al. TRITON-TIMI 38 Investigators: Prasugrel versus clopidogrel in patients with acute coronary syndromes. N Engl J Med 2007; 357: 2001-15.

[25] Wiviott SD, Trenk D, Frelinger AL, et al. PRINCIPLE-TIMI 44 Investigators: Prasugrel compared with high loading- and maintenance-dose clopidogrel in patients with planned percutaneous coronary intervention: the prasugrel in comparison to clopidogrel for inhibition of platelet activation and aggregation-thrombolysis in myocardial infarction 44 trial. Circulation 2007; 116: 2923-2932.

[26] Dangas G, Mehran R, Guagliumi G, et al. HORIZONS-AMI Trial Investigators: Role of clopidogrel loading dose in patients with ST-segment elevation myocardial infarction undergoing primary angioplasty: results from the HORIZONS-AMI (harmonizing outcomes with revascularization and stents in acute myocardial infarction) trial. J Am Coll Cardiol 2009; 54: 1438-46.

[27] Paraskevas KI, Athyros VG, Briana DD, Kakafika AI, Karagiannis A, Mikhailidis DP. Statins exert multiple beneficial effects on patients undergoing percutaneous revascularization procedures. Curr Drug Targets 2007; 8: 942-951. 